\title{
ADéVol : Adapter Décor et Volume
}

Campagne d'étude 2020

\section{Ophélie Vauxion et Guilhem Chapelin}

\section{(2) OpenEdition \\ Journals}

Édition électronique

URL : https://journals.openedition.org/baefe/2393

DOI : $10.4000 /$ baefe.2393

ISSN : 2732-687X

Éditeur

ResEFE

\section{Référence électronique}

Ophélie Vauxion, Guilhem Chapelin, « ADéVol : Adapter Décor et Volume » [notice archéologique], Bulletin archéologique des Écoles françaises à l'étranger [En ligne], Italie, mis en ligne le 22 mai 2021, consulté le 24 mai 2021. URL : http://journals.openedition.org/baefe/2393 ; DOl : https://doi.org/ $10.4000 /$ baefe.2393

Ce document a été généré automatiquement le 24 mai 2021.

\section{(c) (i) (9)}

Le Bulletin archéologique des Écoles françaises à l'étranger est mise à disposition selon les termes de la Licence Creative Commons Attribution - Pas d'Utilisation Commerciale - Pas de Modification 4.0 International. 


\title{
ADéVol : Adapter Décor et Volume
}

Campagne d'étude 2020

\author{
Ophélie Vauxion et Guilhem Chapelin
}

\section{NOTE DE L'AUTEUR}

Date précise de l'opération : 7 septembre-18 septembre 2020

Autorité nationale présente : Parco archeologico di Pompei - MiC

Composition de l'équipe de terrain : Ophélie Vauxion, chercheure associée au Centre Jean Bérard (USR 3133, CNRS-EFR), archéologue - Toichographologue ; Guilhem Chapelin, Centre Jean Bérard (USR 3133, CNRS-EFR), architecte ; Lorenza Guida, Università degli Studi Suor Orsola Benincasa - Napoli, étudiante.

Partenariats institutionnels : CJB, Parco Archeologico di Pompei

Établissement éditeur : CJB

Établissements porteurs de l'opération : CJB

Remerciements : Le programme ADéVol s'insère dans l'axe 2 « Espaces, économie et artisanat dans les cites vesuviennes", sous-axe "l'artisanat urbain et la vie économique antique à Pompéi » du Centre Jean Bérard et la recherche a été financée grâce aux crédits du ministère de l'Europe et des Affaires étrangères dans le cadre de la Mission archéologique "Italie du Sud». Nous tenons à remercier Florence Monier pour sa relecture, ses corrections et conseils.

Données scientifiques produites :

https://centrejeanberard.cnrs.fr/spip.php?article399\&lang=fr

Chroniques de l'EFR :

https://journals.openedition.org/cefr/4124 


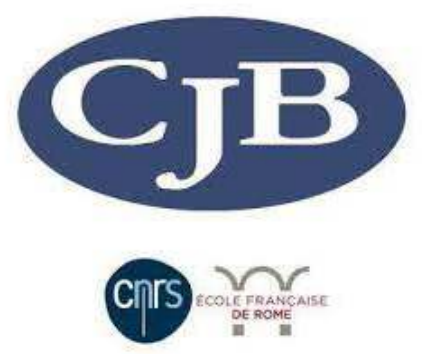

\section{Introduction}

1 La mission ADéVol s'intéresse aux décors peints et stuqués ne possédant pas de zone supérieure, afin de comprendre les spécificités de cette structure bipartite, et ses raisons, architecturales en particulier. Rappelons que notre étude s'appuie sur la classification en « quatre styles pompéiens » établie par A. $\mathrm{Mau}^{1}$, en 1892, à partir des découvertes à Pompéi, et largement discutée depuis lors. Le but de notre recherche est d'améliorer la compréhension et les méthodes de restitution des enduits pariétaux décorés, retrouvés à l'état fragmentaire dans le monde romain, entre le $\mathrm{II}^{\mathrm{e}}$ siècle av. J.-C. et le I ${ }^{\text {er }}$ siècle apr. J.-C. ${ }^{2}$. En 2019, nous avions commencé à cataloguer les compositions décoratives de ce type dans les Regiones I et II de Pompéi.

\section{Activités 2020}

2 Nous avons poursuivi notre enquête dans les Regiones I, VI et IX, ce qui nous a permis d'enregistrer 21 décors dans 14 édifices différents :

- I 3, 28 Casa dei Guerrieri, cubiculum i

- I 3, 29 Casa, triclinium d

- I 13, 16 Casa, triclinium d'été

- I 16, 5 Casa, triclinium 12

- VI 8, 20 Fullonica di L. Veranius Hypsaeus, cubiculum 11

- VI 9, 2-13 Casa di Meleagro, cubiculum 46

- VI 10, 1, Caupona della Via di Mercurio, pièce b et pièce $d$

- VI 15, 7-8 Casa del Principe di Napoli, cubiculum c, tablinium e, cubiculum f et exèdre $m$

- IX 1, 22-29 Casa di M. Epidi Sabini, cubiculum g ${ }^{1}$

- IX 2, 5 Bottega, cubiculum f

- IX 2, 10 Casa del Gallo II, tablinium i

- IX 5, 9 Casa dei Pigmei, pièce l et pièce o

- IX 5, 18 Casa di Jasone, alcôve n et cubiculum $\mathrm{p}$

Comme l'an dernier, tous ces décors ont été cartographiés et intégrés dans la base de données $^{3}$ mise en place sous FileMaker Pro où sont notées toutes les informations concernant l'espace architectural (dimensions, type de sol, fonction) et la composition elle-même (dimensions des zones, largeurs des compartiments, intercompartiments, panneaux, interpanneaux et descriptions du décor par zone). Toutes ces mesures sont prises afin de comprendre les rapports de proportions entre chaque zone. La transition avec le couvrement est, elle aussi, observée et analysée précisément, tout comme la lunette si la pièce est couverte d'une voûte. Sans oublier les ouvertures (portes, 
fenêtres de tous types), et tous les aménagements de la structure des espaces. Chaque décor est décrit; tous les détails techniques pour leur mise en place - raccord des mortiers, journées de travail, tracés préparatoires et même traces accidentelles - ont été soigneusement observés et notés; et ce en lien avec la couverture photographique (ensemble et détails) de chaque paroi.

\section{Résultats et Perspectives}

4 Les premiers résultats avaient permis d'identifier et de caractériser plusieurs spécificités concernant les systèmes décoratifs, l'organisation de la journée de travail et la liaison avec le plafond. Les informations 2020 en confirment certaines et en précisent d'autres.

\section{Les systèmes décoratifs}

5 Sur les 35 décors, sans zone supérieure attestée et recensés depuis 2019, 6 appartiennent au III ${ }^{\text {e }}$ style et les 29 autres sont de IV ${ }^{\text {e }}$ style ${ }^{4}$. Ceci confirme que cette bipartition de la décoration ne peut se retrouver que dans ces deux styles et plus particulièrement dans le $\mathrm{IV}^{\mathrm{e}}$ style. Est-ce que l'on retrouve ces décors de $\mathrm{III}^{\mathrm{e}}$ et $\mathrm{IV}^{\mathrm{e}}$ styles dans les mêmes types d'espace?

6 Les 6 décors de $\mathrm{III}^{\mathrm{e}}$ style proviennent d'édifices des Regiones I et IX, et ornaient des espaces aux fonctions et formes bien différentes:

- I 6, 11 Casa di Casca Longus, atrium ${ }^{5}$

- I 7, 7 Casa del Sacerdos Amandus, cubiculum c

- I 8, 8 Caupona di Lucius Betutius Placidus, cubiculum 6

- I 10, 4 Casa del Menandro, caldarium 48

- I 16, 5 Casa, triclinium 12,

- IX 2, 5 Bottega, cubiculum $\mathrm{f}$

7 Pour les décors de IV ${ }^{\mathrm{e}}$ style, on distingue deux types d'espaces: ceux avec alcôve et ceux sans alcôve. Les pièces avec alcôve sont les moins nombreuses, nous en comptons 5 :

- I 7, 11 Casa dell'Efebo, tablinium 4

- VI 9, 2-13 Casa del Meleagro, cubiculum 46

-VI 15, 7-8 Casa del Principe di Napoli, cubiculum f

- IX 1, 22-29 Casa di Epidi Sabini, cubiculum $\mathrm{g}^{1}$

- IX 5, 18 Casa di Jasone, cubiculum p

Ces pièces possèdent une alcôve sans zone supérieure et, sur les autres parois, un décor un peu plus haut avec une zone supérieure. Ceci concerne principalement des cubicula mais aussi un tablinum. C'est le cas par exemple du cubiculum $\mathrm{f}$ de la Casa del Principe di Napoli (VI 15, 7-8) (fig. 1). Les pièces sans alcôves, plus nombreuses puisque nous en avons recensés 23 , ne semblent pas appartenir à une seule catégorie de pièces. En effet, il s'agit de cubiculum, de tablinium, de triclinium, de vestibule, de latrine, sans compter des pièces sans fonction définie. En couvrement de ces espaces se trouve des voûtes et des plafonds. Les voûtes prennent plusieurs formes : en plein cintre, surbaissée ou avec des plates-bandes. Il n'y a donc pas de couvrement particulier pour ces décors bipartites. 
Fig. 1. Casa del Principe di Napoli (VI 15, 7-8), paroi ouest du cubiculum f.

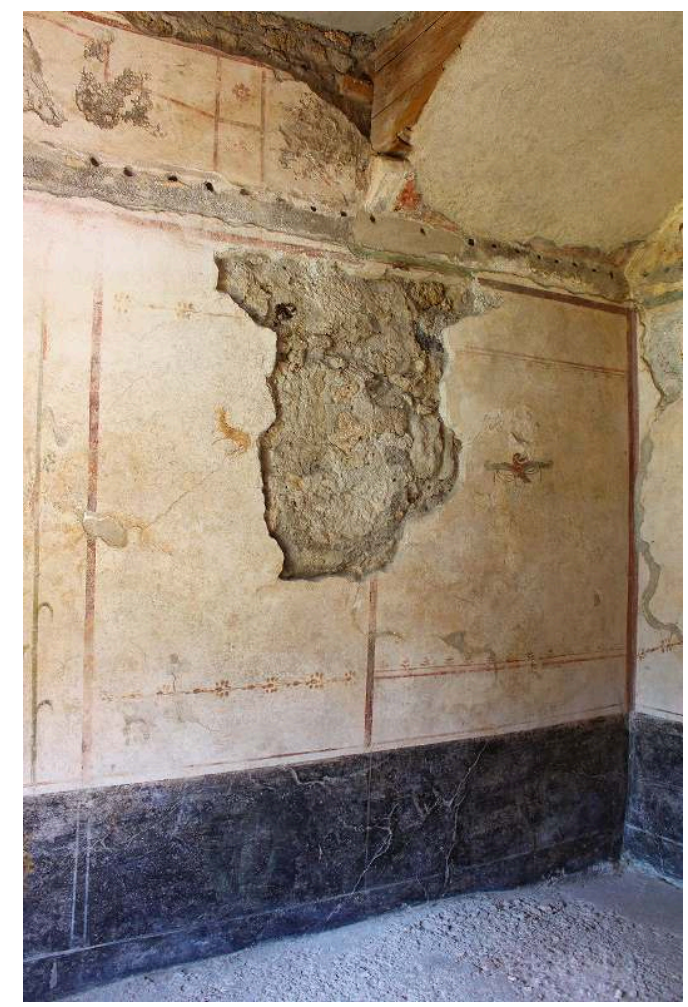

L. Guida, sur concession du MIC - PA Pompei. Tous droits réservés.

Le critère caractéristique entre les décors sans zone supérieure et l'espace est indéniablement la hauteur de paroi qui semble comprise entre 200 et $300 \mathrm{~cm}$. Pourtant, on note deux décors au système décoratif identique dont l'un possède une zone supérieure : le cubiculum 46 de la Casa di Meleagro (VI 9, 2-13) mesure $231 \mathrm{~cm}$ de hauteur jusqu'au départ de la voûte (fig. 2) et le cubiculum c de la Casa del Principe di Napoli (VI 15, 7-8) mesure $228 \mathrm{~cm}$ de hauteur jusqu'à la zone supérieure (fig. 3). Dans le second cas, le décor est peint dans une pièce ayant reçu un premier décor en $I^{\text {er }}$ style dont sont conservées la corniche en stuc et la zone supérieure blanche. La hauteur totale des zones inférieure et principale de ces deux compositions est quasiment identique. Cela nous amène à nous poser indéniablement la question de la place de la zone supérieure dans les décors de $\mathrm{III}^{\mathrm{e}}$ et $\mathrm{IV}^{\mathrm{e}}$ style. Si au III ${ }^{\mathrm{e}}$ style, elle apparaitt très souvent encore liée à la zone principale, cela semble moins le cas avec le IV ${ }^{e}$ style surtout lorsqu'il y a une corniche en stuc qui les séparent. On a l'impression que la zone supérieure n'est là que pour combler un espace préexistant. 
Fig. 02. Casa di Meleagro (VI 9, 2-13), paroi sud du cubiculum 46.

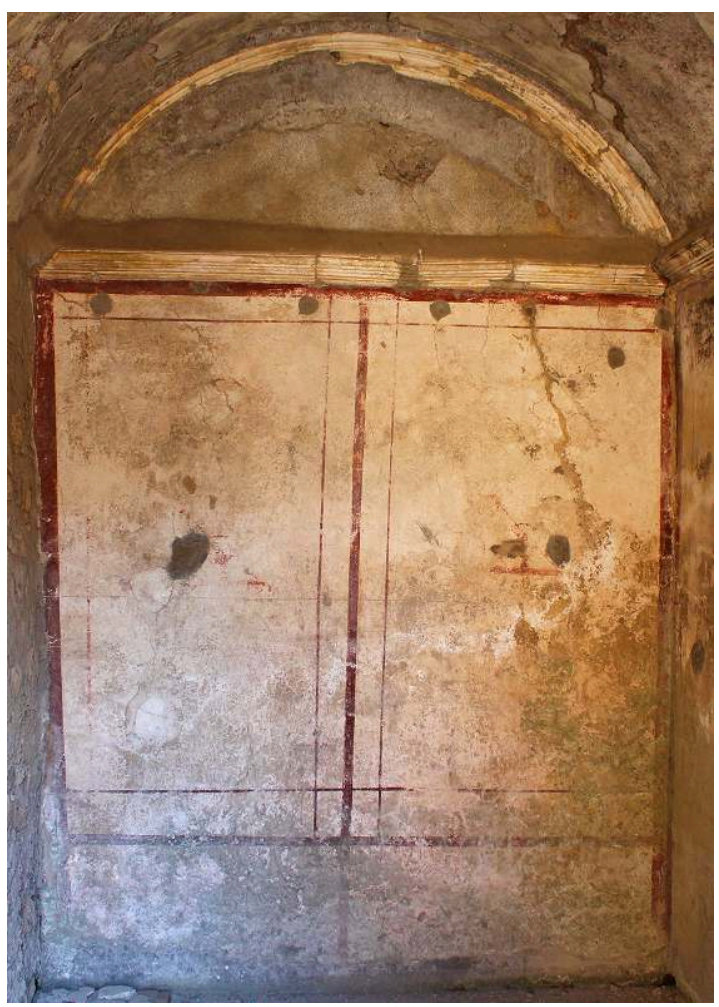

L. Guida, sur concession du MIC - PA Pompei. Tous droits réservés.

Fig. 3. Casa del Principe di Napoli (VI 15, 7-8), paroi est du cubiculum c.

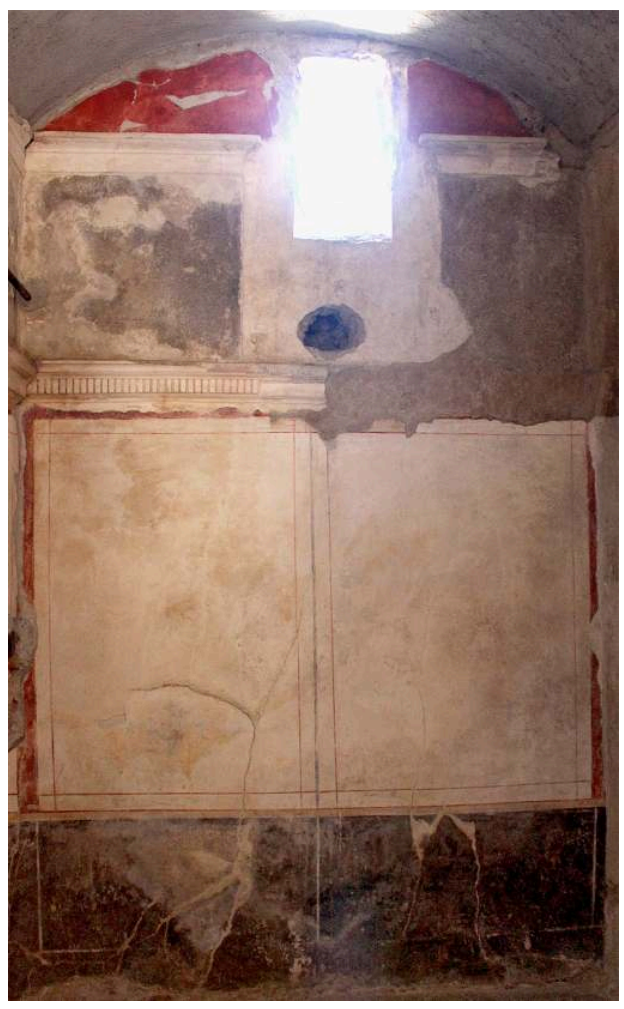

L. Guida, sur concession du MIC - PA Pompei. Tous droits réservés. 


\section{Les aspects techniques}

Cette année encore, nous avons pu constater que ces décors sans zone supérieure sont exécutés rapidement et qu'il n'y a pas systématiquement de journée de travail entre la zone inférieure et la zone principale. Dans le cas de décor un peu plus complexe avec des scènes figurées, l'exécution est rapide mais maîtrisée : les peintres ne prennent pas forcément le temps de masquer les traces laissées dans l'enduit frais. C'est le cas dans la pièce d de la Caupona della via di Mercurio (VI, 10 1) (fig. 4). On observe de nombreuses traces d'ongles sous la prédelle (fig. 5), mais aussi à proximité des amours volants au centre des panneaux. On y voit aussi des tracés préparatoires incisés (fig. 6) et surtout l'utilisation d'une sorte de règle qui sert à laisser une légère empreinte dans l'enduit frais pour positionner au centre la tige végétale de séparation entre les panneaux (fig. 7).

Fig. 04. Caupona della via di Mercurio (VI 10, 1), paroi sud de la pièce d.

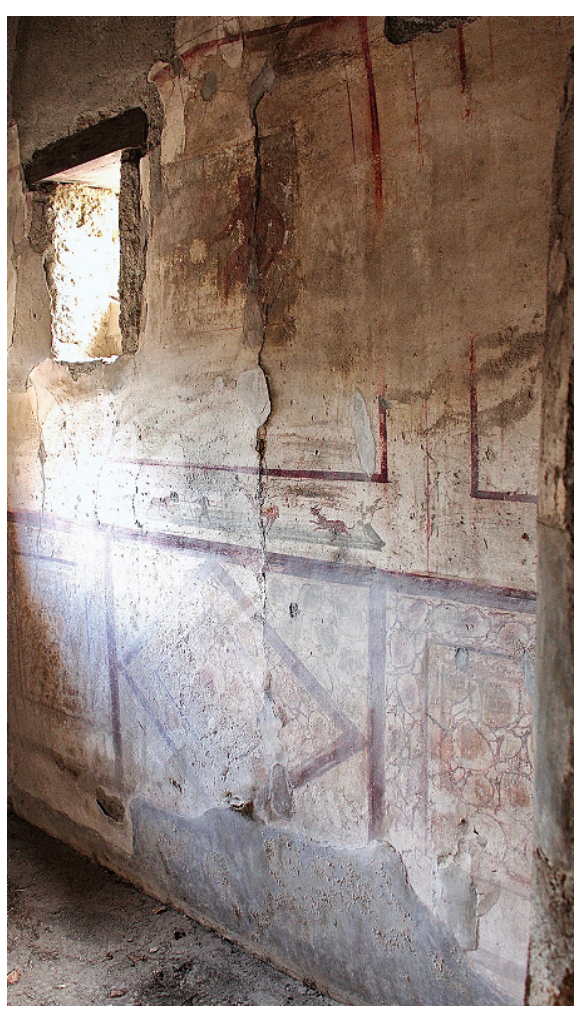

L. Guida, sur concession du MIC - PA Pompei. Tous droits réservés. 
Fig. 5. Caupona della via di Mercurio (VI 10, 1), paroi sud de la pièce d, détail des traces d'ongles sous les personnages de la prédelle.

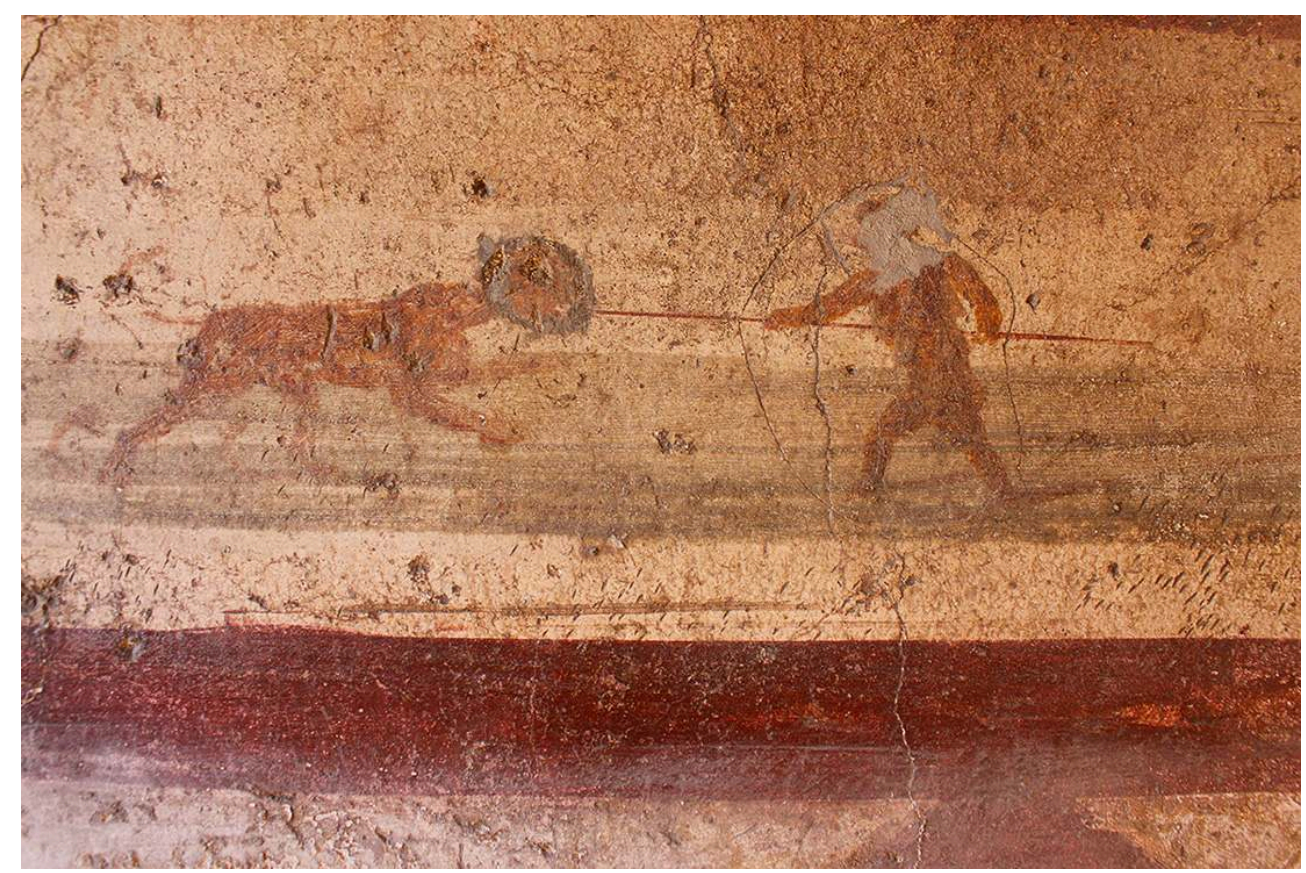

L. Guida, sur concession du MIC - PA Pompei. Tous droits réservés.

Fig. 6. Caupona della via di Mercurio (VI 10, 1), paroi sud de la pièce d, détail du tracé incisé dans le visage de l'amour situé au centre du panneau droit.

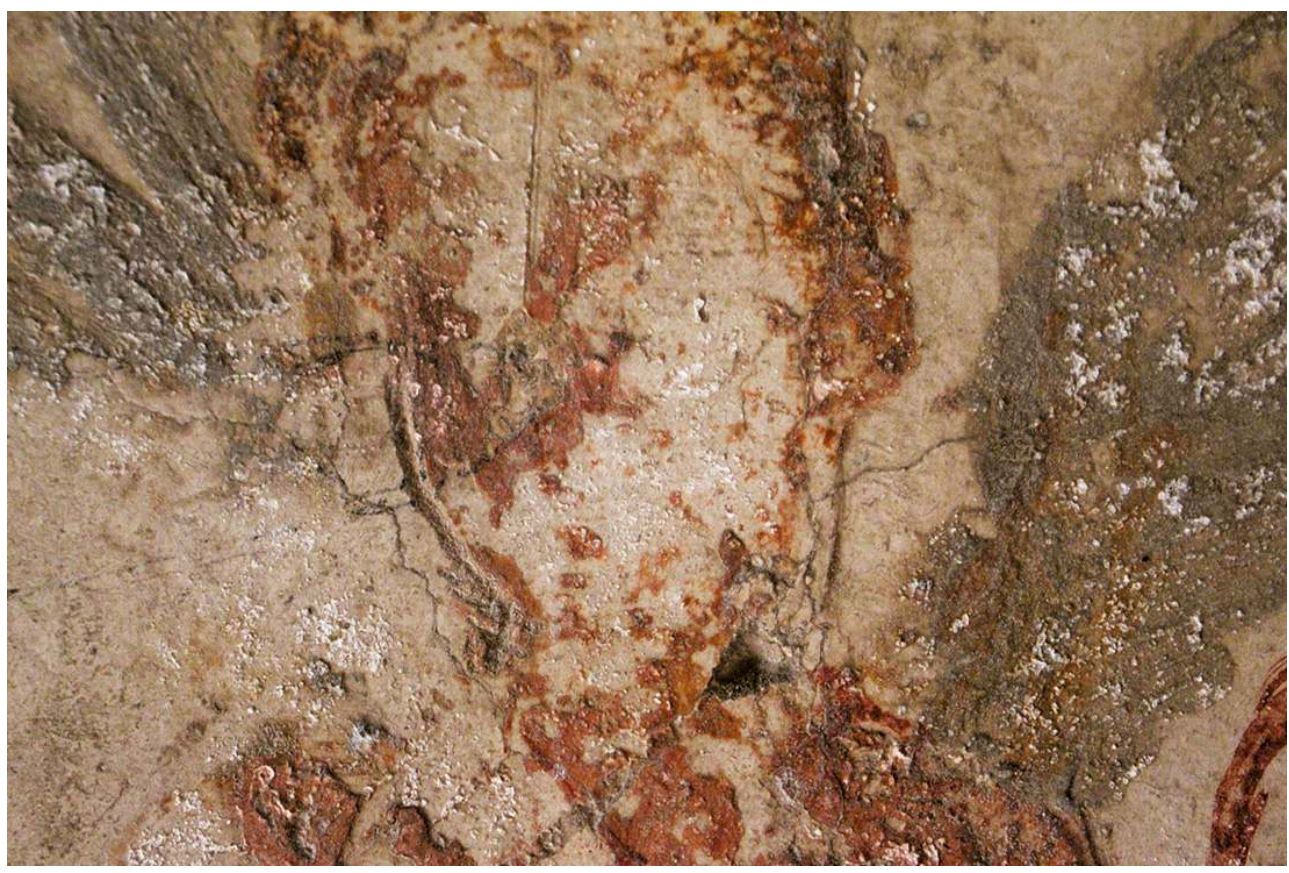

L. Guida, sur concession du MIC - PA Pompei. Tous droits réservés. 
Fig. 7. Caupona della via di Mercurio (VI 10, 1), paroi sud de la pièce $\mathrm{d}$, empreinte d'une règle pour le positionnement de la tige végétale.

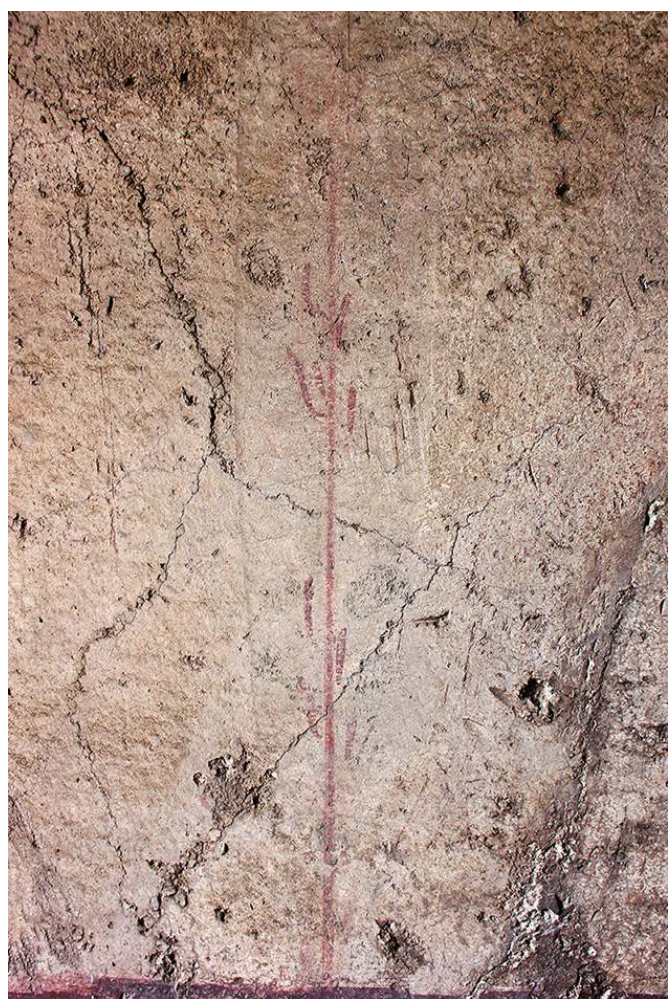

L. Guida, sur concession du MIC - PA Pompei. Tous droits réservés.

11 L'angle dit en sifflet, légèrement aplati et rebondi, n'apparait pas être caractéristique uniquement de la jonction avec un plafond. En effet, nous l'observons sur plusieurs décors en lien avec une corniche en stuc et le départ d'une voûte. De plus dans le péristyle de la Casa del Menandro (I 10,8), cet angle se situe entre la zone principale et la zone supérieure (fig. 8). Il semble dû à la nécessité de bien faire tenir le mortier en appuyant plus fortement. Ce qui est certain, c'est qu'il se trouve toujours au départ d'une zone, maintenant reste à comprendre s'il s'agit constamment de la zone principale ou si c'est aussi le cas pour la zone inférieure. 
Fig. 8. Casa del Menandro $(10,8)$, paroi est du péristyle, détail de l'angle dit en sifflet en haut de la zone principale.

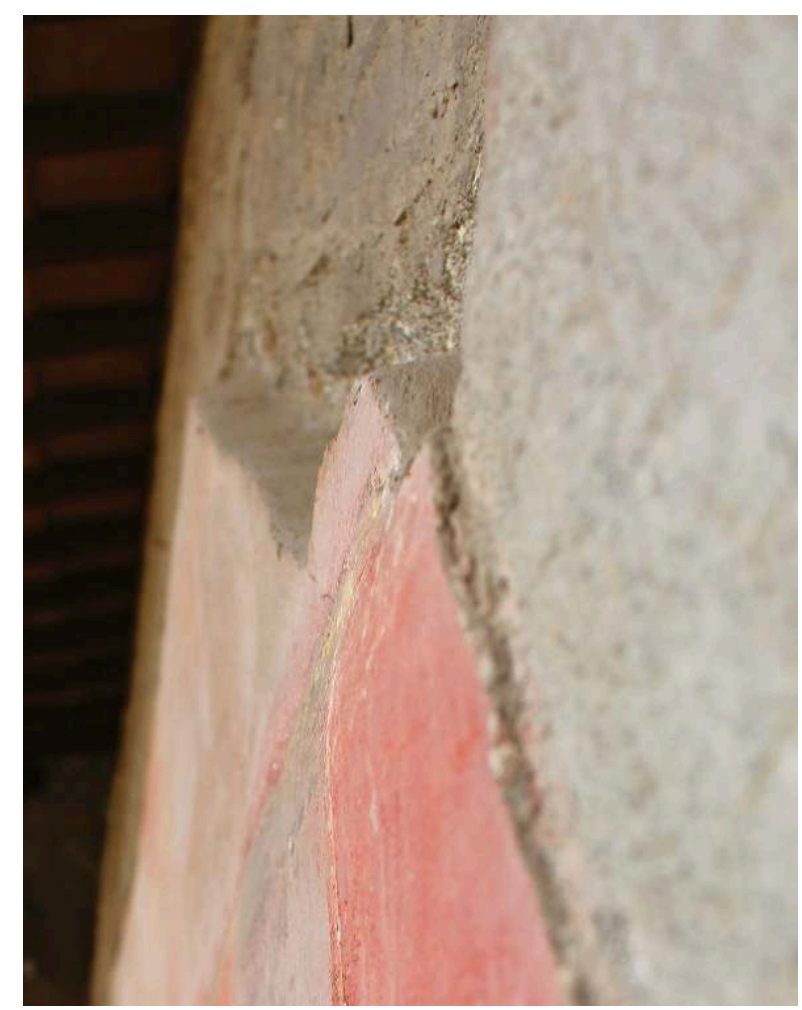

L. Guida, sur concession du MIC - PA Pompei. Tous droits réservés.

Lors de notre enquête nous nous sommes intéressés au triclinium d de la Casa VI 13, 13, pensant que son décor ne possédait pas de zone supérieure or après vérification in situ ce n'est pas le cas. Toutefois, ce décor présente un aspect technique intéressant. L'état de dégradation permet de voir la couche de mortier intermédiaire qui est recouverte ensuite par la couche de surface peinte. Dans cette couche intermédiaire on observe deux lignes parallèles incisées sur toute la longueur de la paroi au niveau de la transition entre la zone inférieure et la zone principale (fig. 9). Il apparaît évident qu'il s'agit de tracés préparatoires indiquant la position de la pontata à venir entre la zone principale et la zone inférieure. 
Fig. 9. Casa (VI 13, 13), paroi est du triclinium d, tracés incisés dans la couche de mortier intermédiaire.

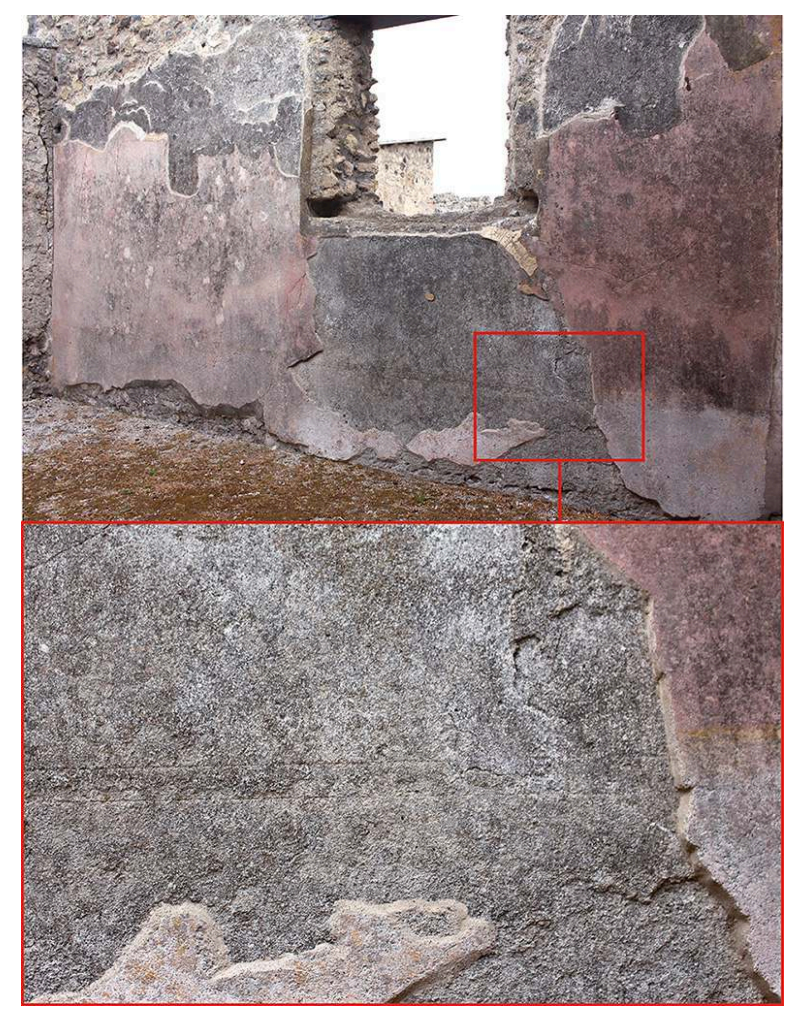

L. Guida, sur concession du MIC - PA Pompei. Tous droits réservés.

\section{Étude des microreliefs pariétaux}

avec la collaboration de G. Chapelin

Nous avons cherché à mettre en évidence des variations de la surface de la peinture à peine sensibles dans une carte de profondeur. Pour ce premier essai nous avons choisi la paroi est de l'exèdre $m$ dans la Casa del Principe di Napoli (VI 15, 7-8). Il a été réalisé un nuage de points dense par photogrammétrie (logiciel Agisoft Metashape) (fig. 10) qui a été comparé (logiciel CloudCompare Open Source Project) à un plan parallèle à la paroi donc quasiment vertical. Après plusieurs essais d'échelles de couleurs il s'est avéré que, pour une meilleure lisibilité, il fallait préférer une répartition sur seulement deux tons. Dans l'image générée (fig. 11) le bleu matérialise les zones les plus proches de l'observateur et le blanc les plus éloignées. Les valeurs limites de cette échelle entre les deux tons ont été resserrées de manière à encadrer les deux centimètres de distances entre le plan et le nuage de points dans lesquels se situent les zones de la paroi qui révèlent le plus d'anomalies. Il apparaît ainsi certaines bandes horizontales ou verticales, correspondantes au décor, qui sont plus claires que le fond et donc légèrement en creux. Cette méthode est encore en cours de développement et il est difficile d'identifier clairement la nature de ces micro-enfoncements. Ils se situent principalement en zone inférieure au niveau des bandes constituant les compartiments et au niveau des bordures ajourées qui les ornent. Au centre de la paroi ils s'observent au niveau de la bordure ajourée du panneau central et des cornes d'abondance qui supportent la bande d'encadrement du panneau. Enfin, on note que le tableautin avec 
les amours est en relief probablement parce qu'il a été inséré après exécution de la fresque.

Fig. 10. Casa del Principe di Napoli (VI 15, 7-8), photogrammétrie de la paroi est de l'exèdre $\mathrm{m}$.

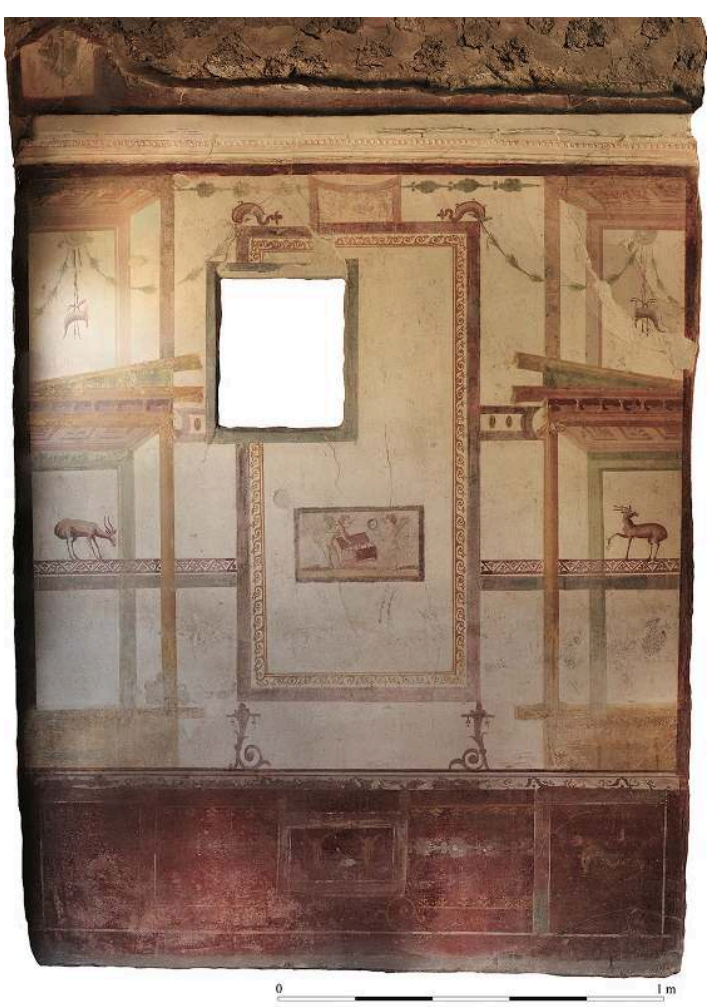

G. Chapelin, sur concession du MIC - PA Pompei. Tous droits réservés. 
Fig. 11. Casa del Principe di Napoli (VI 15, 7-8), carte de profondeur générée par CloudCompare de la paroi est de l'exèdre $\mathrm{m}$.

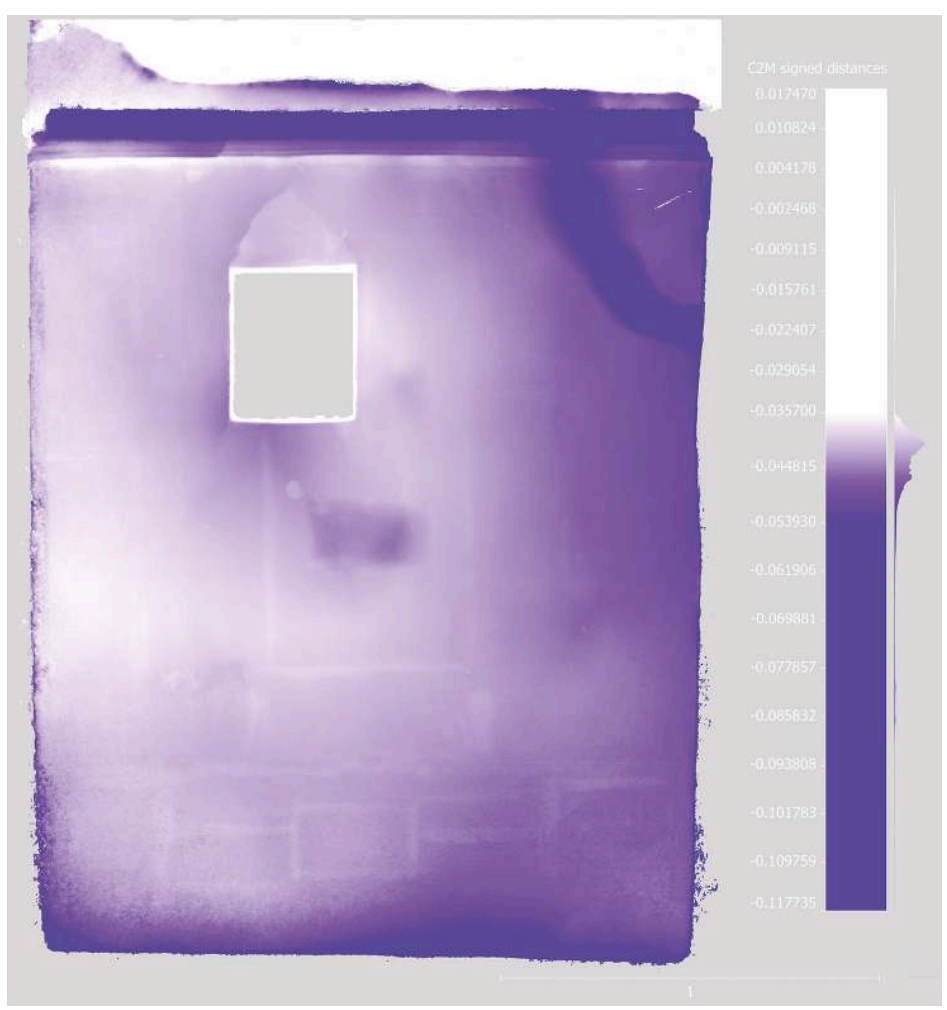

G. Chapelin, sur concession du MIC - PA Pompei. Tous droits réservés.

14 Afin d'améliorer cette méthode, il nous faut faire de nouveau essai en particulier sur des parois où des traces de travail sont bien visible comme sur le pied droit extérieur de l'exèdre $m$ de la Casa del Principe di Napoli (fig. 12). Pour compléter notre étude nous allons faire une campagne de photographies multi-spectrales et la mettre en relation avec l'image générée par le CloudCompare et essayer de comprendre ce que cela nous révèle des méthodes de travail des artisans peintres. 
Fig. 12. Casa del Principe di Napoli (VI 15, 7-8), détail du piedroit extérieur est de l'exèdre $\mathrm{m}$, donnant sur le portique $\mathrm{I}$.

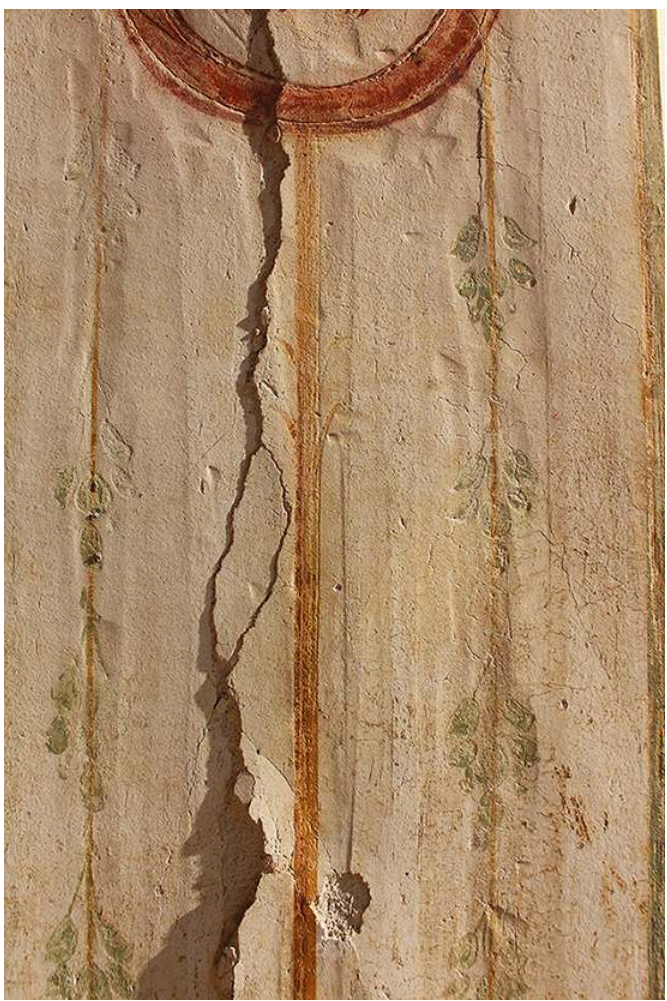

L. Guida sur concession du MIC - PA Pompei. Tous droits réservés.

\section{Perspectives}

Grâce aux campagnes 2019 et 2020, nous avons catalogué presque la moitié des décors sans zone supérieure de Pompéi recensé dans les $P P M^{6}$. Devant l'importance des résultats et des questionnements qui en découlent, pour 2021 nous programmons de continuer cet inventaire dans les Regiones III et VIII et de terminer les régions commencées l'an dernier et cette année si nous obtenons l'autorisation d'accéder aux édifices.

\section{BIBLIOGRAPHIE}

MAU 1882

August Mau, Geschichte der decorativen Wandmalerei in Pompeji, Berlin, G. Reimer, 1882.

VAUXION 2020

Ophélie Vauxion, «ADéVol : Adapter Décor et Volume. Campagne d'étude 2019 », Chronique des activités archéologiques de l'École française de Rome 2020, en ligne, http://journals.openedition.org/ cefr/4124, consulté le 15 janvier 2021. 
PPM 1990

Pompei: pitture e mosaici, Rome, Istituto dell'Enciclopedia Italiana, 1990.

\section{NOTES}

1. Mau 1882.

2. Pour en savoir plus voir Vauxion 2020.

3. Voir Vauxion 2020, fig. 7.

4. Les décors de $\mathrm{III}^{\mathrm{e}}$ style sont datés entre 15 av. J.-C. et 45 apr. J.-C. et les décors de IV ${ }^{\mathrm{e}}$ style entre 45 et 79 apr. J.-C.

5. Pour ce décor l'absence de zone supérieure demande à être confirmée après consultation des archives.

6. PPM 1990.

\section{INDEX}

sujets https://ark.frantiq.fr/ark:/26678/crtJOWlLcbTmc, https://ark.frantiq.fr/ark:/26678/ pcrt5f9rBtTOSr, 26678/pcrtKJVpuP3AET, https://ark.frantiq.fr/ark:/26678/pcrtWegewfItfX, https://ark.frantiq.fr/ark:/26678/pcrtJVKn9XsZH9

Thèmes : $\mathrm{CJB}$

chronologie https://ark.frantiq.fr/ark:/26678/pcrtZTmusVUU24

lieux https://ark.frantiq.fr/ark:/26678/pcrt7ya9wo0Xhb

Année de l'opération : 2020

\section{AUTEURS}

\section{OPHÉLIE VAUXION}

chercheure associée au Centre Jean Bérard (USR 3133, CNRS-EFR)

\section{GUILHEM CHAPELIN}

Centre Jean Bérard (USR 3133, CNRS-EFR) 